\title{
Weighted hypergeometric functions and fractional derivative
}

\author{
JE Restrepo ${ }^{1}$, A Kıllıcman $^{2 *}$, P Agarwal ${ }^{3}$ and Omer Altun²
}

${ }^{*}$ Correspondence: akilic@upm.edu.my

${ }^{2}$ Department of Mathematics, University Putra Malaysia, Serdang, Selangor 43400 UPM, Malaysia Full list of author information is available at the end of the article

\begin{abstract}
We introduce some weighted hypergeometric functions and the suitable generalization of the Caputo fractional derivation. For these hypergeometric functions, some linear and bilinear relations are obtained by means of the mentioned derivation operator. Then some of the considered hypergeometric functions are determined in terms of the generalized Mittag-Leffler function $E_{\left(\rho_{j}\right), \lambda}^{\left(\gamma_{j},()_{j}\right)}\left[z_{1}, \ldots, z_{r}\right]$ (Mainardi in Fractional Calculus and Waves in Linear Viscoelasticity: An Introduction to Mathematical Models, 2010) and the generalized polynomials $S_{n}^{m}[x]$ (Srivastava in Indian J. Math. 14:1-6, 1972). The boundary behavior of some other class of weighted hypergeometric functions is described in terms of Frostman's $\alpha$-capacity. Finally, an application is given using our fractional operator in the problem of fractional calculus of variations.
\end{abstract}

MSC: 30A10; 33E12; 26D07

Keywords: weighted Caputo fractional derivative; weighted hypergeometric function; generating function; Srivastava polynomials; generalized Mittag-Leffler function; $\alpha$-capacity

\section{Introduction}

Recently, many specialists have investigated special functions due to the important role of these functions in mathematical, physical and engineering problems. Various extensions of some special functions were studied in many works (see, e.g., [3-6]).

For introducing some new weighted hypergeometric functions, we use the weighted extension of Euler's beta-function (a particular case associated with the extensions considered in [7]):

$$
B_{\omega}^{(\alpha, \beta)}(x, y)=\int_{0}^{1} t^{x-1}(1-t)^{y-1} \omega^{(\alpha, \beta)}(z t, u, v) d t
$$

where $\operatorname{Re} x>0$, $\operatorname{Re} y>0$ and $\alpha, \beta, z, u, v$ are real or complex parameters and $\omega^{(\alpha, \beta)}(z t, u, v)$ is a function of the class $\Omega$, i.e. such that the integral (1.1) is absolutely convergent. Besides, by writing $B_{\omega}(x, y)$ we mean that this function and the function $\omega$ do not depend on $\alpha$ and $\beta$. One can see that, if $\omega(t, p, 0)=e^{\frac{-p}{t(1-t)}}$ with $\operatorname{Re} p>0$, then $B_{\omega}(x, y)(\min \{\operatorname{Re} x, \operatorname{Re} y\}>0)$ becomes the extension of Euler's beta-function considered by Chaudhry et al. [8]. Besides,

(c) The Author(s) 2017. This article is distributed under the terms of the Creative Commons Attribution 4.0 International License (http://creativecommons.org/licenses/by/4.0/), which permits unrestricted use, distribution, and reproduction in any medium, provided you give appropriate credit to the original author(s) and the source, provide a link to the Creative Commons license, and indicate if changes were made. 
by a straightforward calculation, it follows in the general case that

$$
B_{\omega}(x, y+1)+B_{\omega}(x+1, y)=B_{\omega}(x, y), \quad \omega \in \Omega .
$$

In the last years, the interest to application of the fractional derivative operators a considerable growth. Numerous publications have been devoted to the solutions of different problems by applying these operators (see [9-11]).

Recall that the well-known Caputo fractional derivative is defined as

$$
D_{z}^{\mu} f(z):=\frac{1}{\Gamma(m-\mu)} \int_{0}^{z}(z-t)^{m-\mu-1} \frac{d^{m}}{d t^{m}} f(t) d t
$$

where $m-1<\operatorname{Re} \mu<m, m \in \mathbb{N}$ (the set of positive integers). Here and in the following, let $\mathbb{C}, \mathbb{R}, \mathbb{N}$, and $\mathbb{Z}_{0}^{-}$be the sets of complex numbers, real numbers, positive integers, and non-positive integers, respectively. We introduce the general Caputo fractional derivative suitable with the function (1.1) by the following generalization of that defined in $[12,13]$ :

$$
D_{z}^{\mu, \tau} f(z):=\frac{1}{\Gamma(m-\mu)} \int_{0}^{z}(z-t)^{m-\mu-1} \tau(t, u, v) \frac{d^{m}}{d t^{m}} f(t) d t,
$$

where $m-1<\operatorname{Re} \mu<m, m \in \mathbb{N}$ and $u, v$ are real or complex parameters and $\tau$ is assumed to be a function of the class $\Lambda$, i.e. such that the integral (1.2) is absolutely convergent. It is noted that (1.2) becomes the extended Caputo fractional derivative [12] when $\tau(t, p, z)=$ $e^{\frac{-p z^{2}}{(t z-t)}}(\operatorname{Re} p>0)$, while it becomes the classical Caputo fractional derivative when $\tau \equiv 1$.

\section{Weighted hypergeometric functions}

In the same form as defined in [12], we introduce some weighted versions of the Gauss hypergeometric function ${ }_{2} F_{1}$, the Appell hypergeometric functions $F_{1}, F_{2}$ (see [14]), the Lauricella hypergeometric function $F_{D ; \omega}^{3}$, the generalized Gauss hypergeometric function $F_{\omega}$ and the generalized confluent hypergeometric function ${ }_{1} F_{1}^{\omega}$. Everywhere in this paper we assume that $m \in \mathbb{N}, \omega \in \Omega$ and $B(x, y)(\min \{\operatorname{Re} x, \operatorname{Re} y\}>0)$ is the classical Beta function.

Definition 2.1 The $\omega$-weighted extended Gauss hypergeometric function is

$$
{ }_{2} F_{1}(a, b ; c ; z ; \omega):=\sum_{n=0}^{\infty} \frac{(a)_{n}(b)_{n}}{(b-m)_{n}} \frac{B_{\omega}(b-m+n, c-b+m)}{B(b-m, c-b+m)} \frac{z^{n}}{n !}
$$

where $|z|<1$ and $m<\operatorname{Re} b<\operatorname{Re} c$. For an illustration of the Gauss hypergeometric function see $[15,16]$.

Definition 2.2 The $\omega$-weighted extended Appell hypergeometric function $F_{1}$ is

$$
F_{1}(a, b, c ; d ; x, y ; \omega):=\sum_{n, k=0}^{\infty} \frac{(a)_{n+k}(b)_{n}(c)_{k}}{(a-m)_{n+k}} \frac{B_{\omega}(a-m+n+k, d-a+m)}{B(a-m, d-a+m)} \frac{x^{n}}{n !} \frac{y^{k}}{k !},
$$

where $|x|<1,|y|<1$ and $m<\operatorname{Re} a<\operatorname{Re} d$. 
Definition 2.3 The $\omega$-weighted extended Appell hypergeometric function $F_{2}$ is

$$
\begin{aligned}
& F_{2}(a, b, c ; d, e ; x, y ; \omega) \\
& :=\sum_{n, k=0}^{\infty} \frac{(a)_{n+k}(b)_{n}(c)_{k}}{(b-m)_{n}(c-m)_{k}} \frac{B_{\omega}(b-m+n, d-b+m)}{B(b-m, d-b+m)} \\
& \quad \times \frac{B_{\omega}(c-m+k, e-c+m)}{B(c-m, e-c+m)} \frac{x^{n}}{n !} \frac{y^{k}}{k !}
\end{aligned}
$$

where $|x|+|y|<1, m<\operatorname{Re} b<\operatorname{Re} d$ and $m<\operatorname{Re} c<\operatorname{Re} e$. Besides, we just consider one of the form of Appell hypergeometric function [12, 17].

Definition 2.4 The $\omega$-weighted extended Appell hypergeometric function $F_{D, \omega}^{3}$ is

$$
\begin{aligned}
& F_{D, \omega}^{3}(a, b, c, d ; e ; x, y, z ; \omega) \\
& \quad:=\sum_{n, k, r=0}^{\infty} \frac{(a)_{n+k+r}(b)_{n}(c)_{k}(d)_{r}}{(a-m)_{n+k+r}} \frac{B_{\omega}(a-m+n+k+r, e-a+m)}{B(a-m, e-a+m)} \frac{x^{n}}{n !} \frac{y^{k}}{k !} \frac{z^{r}}{r !},
\end{aligned}
$$

where $\sqrt{|x|}+\sqrt{|y|}+\sqrt{|z|}<1$ and $m<\operatorname{Re} a<\operatorname{Re} e$.

Note that the functions defined above become those in [12] when $\omega(t, p, 0)=e^{\frac{-p}{t(1-t)}}$ and $\operatorname{Re} p>0$. Besides, for $\omega \equiv 1$, these functions reduce to the well-known Gauss hypergeometric function ${ }_{2} F_{1}$, Appell functions $F_{1}, F_{2}$ and Lauricella function $F_{D}^{3}$, respectively.

Definition 2.5 The $\omega$-weighted generalized Gauss hypergeometric function $F_{\omega}$ is

$$
F_{\omega}(a, b ; c ; z)=\sum_{n=0}^{\infty}(a)_{n} \frac{B_{\omega}(b+n, c-b)}{B(b, c-b)} \frac{z^{n}}{n !}
$$

where $|z|<1$ and $\operatorname{Re} c>\operatorname{Re} b>0$.

Definition 2.6 The $\omega$-weighted generalized confluent hypergeometric function ${ }_{1} F_{1}^{\omega}$ is

$$
{ }_{1} F_{1}^{\omega}(b ; c ; z)=\sum_{n=0}^{\infty} \frac{B_{\omega}(b+n, c-b)}{B(b, c-b)} \frac{z^{n}}{n !}
$$

where $|z|<1$ and $\operatorname{Re} c>\operatorname{Re} b>0$.

For some examples of functions $F_{\omega}$ and ${ }_{1} F_{1}^{\omega}$, see [7].

Remark 2.1 If $\omega^{(\alpha, \beta)}(t, p, 0)={ }_{1} F_{1}\left(\alpha ; \beta ; \frac{-p}{t(1-t)}\right)$ where $\min \{\operatorname{Re} x, \operatorname{Re} y, \operatorname{Re} \alpha, \operatorname{Re} \beta\}>0$ and $\operatorname{Re} p \geq 0$, then $B_{\omega}^{(\alpha, \beta)}(x, y)$ is the function $B_{p}^{(\alpha, \beta)}(x, y)$ defined in [5] (see p.32 in [5], also, p.1748 in [18]). Hence, $F_{\omega}(a, b ; c ; z)=F_{p}^{(\alpha, \beta)}(a, b ; c ; z)$ and ${ }_{1} F_{1}^{\omega}(b ; c ; z)={ }_{1} F_{1}^{(\alpha, \beta, p)}(b ; c ; z)$ are the same as those in [18], pp.1748-1749 (see also [5], p.39).

The next definition, it will be useful to introduce a general result. 
Definition 2.7 Let $f(z):=\sum_{n=0}^{\infty} a_{n} z^{n}$ and $g(z):=\sum_{n=0}^{\infty} b_{n} z^{n}$ be two power series whose convergence or radii are $R_{f}$ and $R_{g}$, respectively. Then the Hadamard product of $f(z)$ and $g(z)$ is the power series (see $[18,19])$

$$
(f * g)(z):=\sum_{n=0}^{\infty} a_{n} b_{n} z^{n}
$$

whose convergence radius $R$ satisfies the inequality $R_{f} R_{g} \leq R$.

Remark 2.2 The above definitions can be considered with $B_{\omega}^{(\alpha, \beta)}$ instead of $B_{\omega}$.

\section{The weighted Caputo derivative}

Below, we establish some useful statements, where we apply the weighted Caputo fractional derivative. The next results are some generalizations of those in $[12,14,18]$ and some others. Hence, the tools to prove them (lemmas and theorems) are similar.

Lemma 3.1 If $m-1<\operatorname{Re} \mu<m, \omega \in \Lambda, \omega \in \Omega, s, w \in \mathbb{C}$ and $\operatorname{Re} \mu<\operatorname{Re} \lambda$, then

$$
D_{z}^{\mu, \omega}\left[z^{\lambda}\right]=\frac{\Gamma(\lambda+1) B_{\omega}(\lambda-m+1, m-\mu)}{\Gamma(\lambda-\mu+1) B(\lambda-m+1, m-\mu)} z^{\lambda-\mu} .
$$

Proof Indeed,

$$
\begin{aligned}
D_{z}^{\mu, \omega}\left[z^{\lambda}\right] & =\frac{1}{\Gamma(m-\mu)} \int_{0}^{z}(z-t)^{m-\mu-1} \omega(t, s, w) \frac{d^{m}}{d t^{m}} t^{\lambda} d t \\
& =\frac{\Gamma(\lambda+1)}{\Gamma(m-\mu) \Gamma(\lambda-m+1)} \int_{0}^{z}(z-t)^{m-\mu-1} t^{\lambda-m} \omega(t, s, w) d t \\
& =\frac{\Gamma(\lambda+1) z^{\lambda-\mu}}{\Gamma(m-\mu) \Gamma(\lambda-m+1)} \int_{0}^{1}(1-u)^{m-\mu-1} u^{\lambda-m} \omega(z u, s, w) d u \\
& =\frac{\Gamma(\lambda+1) B_{\omega}(\lambda-m+1, m-\mu)}{\Gamma(\lambda-\mu+1) B(\lambda-m+1, m-\mu)} z^{\lambda-\mu} .
\end{aligned}
$$

Theorem 3.1 If $f(z)=\sum_{n=0}^{\infty} a_{n} z^{n}$ is an analytic function in the disk $|z|<\rho, \omega \in \Lambda$ and $\omega \in \Omega$, then

$$
D_{z}^{\mu, \omega}\{f(z)\}=\sum_{n=0}^{\infty} D_{z}^{\mu, \omega}\left\{z^{n}\right\}
$$

where $m-1<\operatorname{Re} \mu<m$.

Proof As the power series converges uniformly, the integral of $D_{z}^{\mu, \omega}\{f(z)\}$ converges absolutely, and hence the desired result follows by a straightforward calculation.

Theorem 3.2 Let $m-1<\operatorname{Re} \lambda-\mu<m<\operatorname{Re} \lambda, \kappa \in \mathbb{C}, \omega \in \Lambda$ and $\omega \in \Omega$. Then

$$
D_{z}^{\lambda-\mu, \omega}\left\{z^{\lambda-1}(1-z)^{-\kappa}\right\}=z^{\mu-1} \frac{\Gamma(\lambda)}{\Gamma(\mu)}{ }_{2} F_{1}(\kappa, \lambda ; \mu ; z ; \omega), \quad|z|<1 .
$$


Proof Using the power series of $(1-z)^{-\kappa}$, Theorem 3.1, Lemma 3.1 and equation (2.1), we obtain

$$
\begin{aligned}
D_{z}^{\lambda-\mu, \omega}\left\{z^{\lambda-1}(1-z)^{-\kappa}\right\} & =D_{z}^{\lambda-\mu, \omega}\left\{z^{\lambda-1} \sum_{n=0}^{\infty}(\kappa)_{n} \frac{z^{n}}{n !}\right\} \\
& =\sum_{n=0}^{\infty} \frac{(\kappa)_{n}}{n !} D_{z}^{\lambda-\mu, \omega}\left\{z^{\lambda+n-1}\right\} \\
& =z^{\mu-1} \sum_{n=0}^{\infty} \frac{(\kappa)_{n} \Gamma(\lambda+n) B_{\omega}(\lambda-m+n, m-\lambda+\mu)}{\Gamma(\mu+n) B(\lambda-m+n, m-\lambda+\mu)} \frac{z^{n}}{n !} \\
& =z^{\mu-1} \frac{\Gamma(\lambda)}{\Gamma(\mu)} \sum_{n=0}^{\infty} \frac{(\kappa)_{n}(\lambda)_{n}}{(\lambda-m)_{n}} \frac{B_{\omega}(\lambda-m+n, m-\lambda+\mu)}{B(\lambda-m, \mu-\lambda+m)} \frac{z^{n}}{n !} \\
& =z^{\mu-1} \frac{\Gamma(\lambda)}{\Gamma(\mu)}{ }_{2} F_{1}(\kappa, \lambda ; \mu ; z, \omega) .
\end{aligned}
$$

Theorem 3.3 If $m-1<\operatorname{Re}(\lambda-\mu)<m<\operatorname{Re} \lambda, \omega \in \Lambda$ and $\omega \in \Omega$, then

$$
D_{z}^{\lambda-\mu, \omega}\left\{z^{\lambda-1}(1-r z)^{-\kappa}(1-s z)^{-\theta}\right\}=z^{\mu-1} \frac{\Gamma(\lambda)}{\Gamma(\mu)} F_{1}(\lambda, \kappa, \theta ; \mu ; r z ; s z ; \omega)
$$

for $r, s, \kappa, \theta \in \mathbb{C},|r z|<1$ and $|s z|<1$.

Proof Using the power series of $(1-r z)^{-\kappa},(1-s z)^{-\theta}$, Theorem 3.1, Lemma 3.1 and (2.2), we get

$$
\begin{aligned}
& D_{z}^{\lambda-\mu, \omega}\left\{z^{\lambda-1}(1-r z)^{-\kappa}(1-s z)^{-\theta}\right\} \\
& =D_{z}^{\lambda-\mu, \omega}\left(\sum_{n, k=0}^{\infty} \frac{(\kappa)_{n}(\theta)_{k} r^{n} s^{k} z^{\lambda+n+k-1}}{n ! k !}\right) \\
& =\sum_{n, k=0}^{\infty} \frac{(\kappa)_{n}(\theta)_{k} r^{n} s^{k}}{n ! k !} D_{z}^{\lambda-\mu, \omega}\left\{z^{\lambda+n+k-1}\right\} \\
& =z^{\mu-1} \sum_{n, k=0}^{\infty} \frac{(\kappa)_{n}(\theta)_{k} r^{n} s^{k}}{n ! k !} \frac{\Gamma(\lambda+n+k) B_{\omega}(\lambda-m+n+k, m-\lambda+\mu)}{\Gamma(\mu+n+k) B(\lambda-m+n+k, m-\lambda+\mu)} z^{n+k} \\
& =z^{\mu-1} \frac{\Gamma(\lambda)}{\Gamma(\mu)} \sum_{n, k=0}^{\infty} \frac{(\lambda)_{n+k}(\kappa)_{n}(\theta)_{k}}{(\lambda-m)_{n+k}} \frac{B_{\omega}(\lambda-m+n+k, m-\lambda+\mu)}{B(\lambda-m, m-\lambda+\mu)} \frac{(r z)^{n}}{n !} \frac{(s z)^{k}}{k !} \\
& =z^{\mu-1} \frac{\Gamma(\lambda)}{\Gamma(\mu)} F_{1}(\lambda, \kappa, \theta ; \mu ; r z ; s z ; \omega) .
\end{aligned}
$$

Theorem 3.4 If $m-1<\operatorname{Re}(\lambda-\mu)<m<\operatorname{Re} \lambda, \omega \in \Lambda, \omega \in \Omega$ and $m<\operatorname{Re} \beta<\operatorname{Re} \gamma$, then

$$
\begin{aligned}
& D_{z}^{\lambda-\mu, \omega}\left(z^{\lambda-1}(1-z)_{2}^{-\alpha} F_{1}\left(\alpha, \beta ; \gamma ; \frac{x}{1-z} ; \omega\right)\right) \\
& =\frac{\Gamma(\lambda)}{\Gamma(\mu)} z^{\mu-1} F_{2}(\alpha, \beta, \lambda, \gamma ; \mu ; x, z ; \omega), \quad|x|+|z|<1 .
\end{aligned}
$$


Proof By the power series of $(1-a z)^{-\alpha},(2.1)$ and (3.1), we obtain

$$
\begin{aligned}
& D_{z}^{\lambda-\mu, \omega}\left(z^{\lambda-1}(1-z)^{-\alpha}{ }_{2} F_{1}\left(\alpha, \beta ; \gamma ; \frac{x}{1-z} ; \omega\right)\right) \\
&=D_{z}^{\lambda-\mu, \omega}\left\{z^{\lambda-1}(1-z)^{-\alpha-n}\right\} \times \sum_{n=0}^{\infty} \frac{(\alpha)_{n}(\beta)_{n}}{(\beta-m)_{n}} \frac{B_{\omega}(\beta-m+n, \gamma-\beta+m)}{B(\beta-m, \gamma-\beta+m)} \frac{x^{n}}{n !} \\
&=z^{\mu-1} \frac{\Gamma(\lambda)}{\Gamma(\mu)} \sum_{n, k=0}^{\infty} \frac{(\alpha)_{n+k}(\beta)_{n}(\lambda)_{k}}{(\beta-m)_{n}(\lambda-m)_{k}} \frac{B_{\omega}(\beta-m+n, \gamma-\beta+m)}{B(\beta-m, \gamma-\beta+m)} \\
& \quad \times \frac{B_{\omega}(\lambda-m+k, \mu-\lambda+m)}{B(\lambda-m, \mu-\lambda+m)} \frac{x^{n}}{n !} \frac{z^{k}}{k !} \\
&= z^{\mu-1} \frac{\Gamma(\lambda)}{\Gamma(\mu)} F_{2}(\lambda, \alpha, \beta ; \mu ; a z ; b z ; \omega) .
\end{aligned}
$$

\section{Generating functions}

Below we obtain some bilinear generating relations for the weighted extended hypergeometric function ${ }_{2} F_{1}$. In a sense, these relations are similar to those in $[7,12]$ by taking some particular weights $\omega$ belonging to $\Lambda \cap \Omega$.

Theorem 4.1 If $m-1<\operatorname{Re}(\lambda-\mu)<\operatorname{Re} \mu, \omega \in \Lambda$ and $\omega \in \Omega$, then

$$
\sum_{n=0}^{\infty} \frac{(\alpha)_{n}}{n !}{ }_{2} F_{1}(\alpha+n, \lambda ; \mu ; z ; \omega) t^{n}=(1-t)^{-\alpha}{ }_{2} F_{1}\left(\alpha, \lambda ; \mu ; \frac{z}{1-t} ; \omega\right)
$$

where $|z|<\min \{1,|1-t|\}$.

Proof Note that by the identity of [12]

$$
[(1-z)-t]^{-\alpha}=(1-t)^{-\alpha}\left(1-\frac{z}{1-t}\right)^{-\alpha}
$$

We write a power series in the left hand side

$$
\sum_{n=0}^{\infty} \frac{(\alpha)_{n}}{n !}(1-z)^{-\alpha}\left(\frac{t}{1-z}\right)^{n}=(1-t)^{-\alpha}\left(1-\frac{z}{1-t}\right)^{-\alpha}
$$

where $|t|<|1-z|$. Besides, by multiplying both sides by $z^{\lambda-1}$ and applying our operator $D_{z}^{\lambda-\mu, \omega}$, we get

$$
D_{z}^{\lambda-\mu, \omega}\left\{\sum_{n=0}^{\infty} \frac{(\alpha)_{n} t^{n}}{n !} z^{\lambda-1}(1-z)^{-\alpha-n}\right\}=D_{z}^{\lambda-\mu, \omega}\left\{(1-t)^{-\alpha} z^{\lambda-1}\left(1-\frac{z}{1-t}\right)^{-\alpha}\right\}
$$

As $|t|<|1-z|$ and $0<\operatorname{Re} \mu<\operatorname{Re} \lambda$, the fractional derivative can be replaced inside the sum:

$$
\sum_{n=0}^{\infty} \frac{(\alpha)_{n}}{n !} D_{z}^{\lambda-\mu, \omega}\left\{z^{\lambda-1}(1-z)^{-\alpha-n}\right\} t^{n}=(1-t)^{-\alpha} D_{z}^{\lambda-\mu, \omega}\left\{z^{\lambda-1}\left(1-\frac{z}{1-t}\right)^{-\alpha}\right\}
$$

Finally, by Theorem 3.2, we get (4.1). 
Theorem 4.2 If $m-1<\operatorname{Re}(\lambda-\mu)<\operatorname{Re} \mu, \omega \in \Lambda$ and $\omega \in \Omega$, then

$$
\sum_{n=0}^{\infty} \frac{(\alpha)_{n}}{n !}{ }_{2} F_{1}(\beta-n, \lambda ; \mu ; z ; \omega) t^{n}=(1-t)^{-\alpha} F_{1}\left(\beta, \alpha, \lambda ; \mu ; z ; \frac{z t}{1-t} ; \omega\right)
$$

where $|t|<\frac{1}{1+|z|}$.

Proof By the identity of [12] we get

$$
[1-(1-z) t]^{-\alpha}=(1-t)^{-\alpha}\left(1+\frac{-z t}{1-t}\right)^{-\alpha}
$$

and we write the power series in the left hand side

$$
\sum_{n=0}^{\infty} \frac{(\alpha)_{n}}{n !}(1-z)^{n} t^{n}=(1-t)^{-\alpha}\left(1-\frac{-z t}{1-t}\right)^{-\alpha}, \quad|t|<1 /|1-z|
$$

Besides, by multiplying both sides by $z^{\lambda-1}(1-z)^{-\beta}$ and applying the weighted Caputo fractional derivative $D_{z}^{\lambda-\mu, \omega}$ we get

$$
D_{z}^{\lambda-\mu, \omega}\left\{\sum_{n=0}^{\infty} \frac{(\alpha)_{n}}{n !} z^{\lambda-1}(1-z)^{-\beta-n} t^{n}\right\}=D_{z}^{\lambda-\mu, \omega}\left\{(1-t)^{-\alpha} z^{\lambda-1}(1-z)^{-\beta}\left(1-\frac{-z t}{1-t}\right)^{-\alpha}\right\}
$$

As $|z t|<|1-t|$ and $0<\operatorname{Re} \mu<\operatorname{Re} \lambda$, the derivative can be replaced inside the sum:

$$
\sum_{n=0}^{\infty} \frac{(\alpha)_{n}}{n !} D_{z}^{\lambda-\mu, \omega}\left\{z^{\lambda-1}(1-z)^{-\alpha-n}\right\} t^{n}=(1-t)^{-\alpha} D_{z}^{\lambda-\mu, \omega}\left\{z^{\lambda-1}(1-z)^{-\beta}\left(1-\frac{-z t}{1-t}\right)^{-\alpha}\right\}
$$

Hence, equation (4.2) follows by Theorems 3.2 and 3.3.

Theorem 4.3 If $m-1<\operatorname{Re}(\beta-\gamma)<\operatorname{Re} \beta, m<\operatorname{Re} \lambda<\operatorname{Re} \mu, \omega \in \Lambda$ and $\omega \in \Omega$, then

$$
\sum_{n=0}^{\infty} \frac{(\alpha)_{n}}{n !}{ }_{2} F_{1}(\alpha+n, \lambda ; \mu ; z ; \omega)_{2} F_{1}(-n, \beta ; \gamma ; u ; \omega)=F_{2}\left(\alpha, \lambda, \beta ; \mu, \gamma ; z, \frac{u t}{1-t} ; \omega\right)
$$

Proof If $t$ tends to $(1-u) t$ in equation (4.1) and multiplying both sides by $u^{\beta-1}$, we obtain

$$
\begin{aligned}
& \sum_{n=0}^{\infty} \frac{(\alpha)_{n}}{n !}{ }_{2} F_{1}(\alpha+n, \lambda ; \mu ; z ; \omega) u^{\beta-1}(1-u)^{n} t^{n} \\
& \quad=u^{\beta-1}[1-(1-u) t]_{2}^{-\alpha} F_{1}\left(\alpha, \lambda ; \mu ; \frac{z}{1-(1-u) t} ; \omega\right) .
\end{aligned}
$$

Hence, applying the fractional derivative $D_{u}^{\beta-\lambda, \omega}$ to both sides we get

$$
\begin{aligned}
& \sum_{n=0}^{\infty} \frac{(\alpha)_{n}}{n !}{ }_{2} F_{1}(\alpha+n, \lambda ; \mu ; z ; \omega) D_{u}^{\beta-\lambda, \omega}\left\{u^{\beta-1}(1-u)^{n}\right\} t^{n} \\
& \quad=D_{u}^{\beta-\lambda, \omega}\left\{u^{\beta-1}[1-(1-u) t]^{-\alpha}{ }_{2} F_{1}\left(\alpha, \lambda ; \mu ; \frac{z}{1-(1-u) t} ; \omega\right)\right\}
\end{aligned}
$$


where $|z|<1,\left|\frac{1-u}{1-z} t\right|<1$ and $\left|\frac{z}{1-t}\right|+\left|\frac{u t}{1-t}\right|<1$. This formula is the same as

$$
\begin{aligned}
& \sum_{n=0}^{\infty} \frac{(\alpha)_{n}}{n !}{ }_{2} F_{1}(\alpha+n, \lambda ; \mu ; z ; \omega) D_{u}^{\beta-\lambda, \omega}\left\{u^{\beta-1}(1-u)^{n}\right\} t^{n} \\
& \quad=D_{u}^{\beta-\lambda, \omega}\left\{u^{\beta-1}\left[1-\frac{-u t}{1-t}\right]^{-\alpha}{ }_{2} F_{1}\left(\alpha, \lambda ; \mu ; \frac{z}{1-\frac{-u t}{1-t}} ; \omega\right)\right\} .
\end{aligned}
$$

Therefore, we obtain the desired statement (4.3) by Theorems 3.2 and 3.4.

Now, using the weighted function $F_{\omega}$, we introduce a generalization of the generating relation given in [18], pp.1750-1751 (see also [14]).

Theorem 4.4 If $\operatorname{Re} c>\operatorname{Re} b>0$ and $\omega \in \Omega$, then

$$
\begin{aligned}
(1+t)^{-\lambda} F_{\omega}(a, b ; c, z /(1+t)) \\
\quad=\sum_{r=0}^{\infty}(-1)^{r}(\lambda)_{r} F_{\omega}(a, b ; c ; z) *{ }_{1} F_{1}(\lambda+r ; \lambda ; z) \frac{t^{r}}{r !}, \quad z, \lambda \in \mathbb{C},|t|<1 .
\end{aligned}
$$

Proof The proof runs parallel to that of Theorem 2.1 in [18]. We omit the details.

Remark 4.1 By Remark 2.2, we can get all results of the sections (the weighted Caputo derivative and Generating functions), in the same way. Besides, these results become those in [12] when we consider the particular $\omega(t, p, 0)=e^{\frac{-p}{t(1-t)}}$ and $\operatorname{Re} p>0$.

\section{Further results and observations}

Note that, if $\omega^{(\alpha, \beta)}(t, p, s)=\frac{\Gamma(\beta)}{\Gamma(\alpha) \Gamma(\beta-\alpha)} \Gamma_{p t^{2}}(s)$, then $B_{\omega}^{(\alpha, \beta)}$ becomes the following generalized gamma function (see [5], pp.32-33):

$$
B_{\omega}^{(\alpha, \beta)}(\alpha-s, \beta-\alpha)=\frac{\Gamma(\beta)}{\Gamma(\alpha) \Gamma(\beta-\alpha)} \int_{0}^{1} t^{\alpha-s-1}(1-t)^{\beta-\alpha-1} \Gamma_{p t^{2}}(s) d t=\Gamma_{p}^{(\alpha, \beta)}(s)
$$

where $\min \{\operatorname{Re} y, \operatorname{Re} \alpha, \operatorname{Re} \beta, \operatorname{Re} p, \operatorname{Re} s\}>0, \Gamma_{p}(s)$ is Chaudhry's gamma function [8] and $\Gamma_{p}^{(\alpha, \beta)}(s)$ is defined in [5], p.33.

Now, we shall write some of the considered weighted functions in terms of the wellknown Mittag-Leffler function [1]. We work with the generalization of the multivariable Mittag-Leffler function $E_{\left(\rho_{j}\right), \lambda}^{\left(\gamma_{j}\right),\left(l_{j}\right)}\left[z_{1}, \ldots, z_{r}\right]$ introduced by Saxena et al. [20], p.547, Eq. (7.1) (see also [21], pp.2-3) and the generalized polynomials $S_{n}^{m}[x]$ (see [2], p.1, Eq. (1)).

Corollary 5.1 If $\omega\left(x_{1}, z_{1}, 0\right)=S_{n_{1}}^{m_{1}}\left[x_{1}^{\lambda_{1}}\left(1-x_{1}\right)^{\eta}\right] \exp \left\{z_{1}\left(x_{1}\right)^{\mu_{1}}\left(1-x_{1}\right)^{\delta_{1}}\right\}$ and $\eta, \lambda_{1}, \delta_{1}, \mu_{1} \in \mathbb{C}$; $j \geq 0, \operatorname{Re}\left(a-m+n+k+\lambda_{1} j+\mu_{1} k_{1}\right)>0 ; \operatorname{Re}\left(d-a+m+n j+\delta_{1} k_{1}\right)>0$, then

$$
\begin{aligned}
& F_{1}(a, b, c ; d ; x, y ; \omega) \\
& =\sum_{n, k=0}^{\infty} \sum_{j=0}^{\left[n_{1} / m_{1}\right]} \frac{(a)_{n+k}(b)_{n}(c)_{k}}{(a-m)_{n+k}} \frac{\Gamma(d) \Gamma\left(a-m+n+k+\lambda_{1} j\right) \Gamma(d-a+m+\lambda j)}{\Gamma(a-m) \Gamma(d-a+m)} \\
& \quad \times \frac{\left(-n_{1}\right)_{m_{1} j}}{j !} A_{n_{1}, j} E_{\left(1,\left(\mu_{1}+\delta_{1}\right)\right), 1, d-a+m+\eta j+\left(a-m+n+k+\lambda_{1} j\right)}^{\left(1, a-m+n+k+\lambda_{1} j, d-a+m+\eta j\right)\left(1, \mu_{1}, \delta_{1}\right)}\left[z_{1}\right] \frac{x^{n}}{n !} \frac{y^{k}}{k !},
\end{aligned}
$$

where $|x|<1,|y|<1$ and $m<\operatorname{Re} a<\operatorname{Re} d$. 
Proof The desired equality holds by Corollary 3.13 in [21] (p.13) and Definition 2.2.

Corollary 5.2 If $\omega\left(x_{1}, z_{1}, 0\right)=S_{n_{1}}^{m_{1}}\left[x_{1}^{\lambda_{1}}\left(1-x_{1}\right)^{\eta}\right] E_{\left(\rho_{1}\right), \lambda}^{\left(\gamma_{1}\right),\left(l_{1}\right)}\left[z_{1}\left(x_{1}\right)^{\mu_{1}}\left(1-x_{1}\right)^{\delta_{1}}\right]$ and $\eta, \lambda, \lambda_{1}, \gamma_{1}, \rho_{1}$, $\delta_{1}, \mu_{1} \in \mathbb{C} ; \operatorname{Re} \rho_{1}>0 ; \operatorname{Re} \gamma_{1}>0 ; j \geq 0, \operatorname{Re}\left(a-m+n+k+r+\lambda_{1} j+\mu_{1} k_{1}\right)>0 ; \operatorname{Re}(e-a+m+$ $\left.\eta j+\delta_{1} k_{1}\right)>0 ; l_{1} \in \mathbb{N} ; \lambda \notin \mathbb{Z}_{0}^{-}$, then

$$
\begin{aligned}
& F_{D, \omega}^{3}(a, b, c, d ; e ; x, y, z ; \omega) \\
& =\sum_{n, k, r=0}^{\infty} \sum_{j=0}^{\left[n_{1} / m_{1}\right]} \frac{\left(-n_{1}\right)_{m_{1} j}}{j !} \frac{(a)_{n+k+r}(b)_{n}(c)_{k}(d)_{r}}{(a-m)_{n+k+r}} \frac{\Gamma(e) \Gamma\left(a-m+n+k+r+\lambda_{1} j\right)}{\Gamma(a-m) \Gamma(e-a+m)} \\
& \quad \times \Gamma(e-a+m+\lambda j) A_{n_{1}, j} E_{\left(\rho_{1},\left(\mu_{1}+\delta_{1}\right)\right), \lambda, e-a+m+n_{1} j+\left(a-m+n+k+r+\lambda_{1}\right)}^{\left(\gamma_{1}, a-m+n+k+\lambda_{1} j, e-a+m\right),\left(l_{1}, \mu_{1}, \delta_{1}\right)}\left[z_{1}\right] \frac{x^{n}}{n !} \frac{y^{k}}{k !} \frac{z^{r}}{r !},
\end{aligned}
$$

where $\sqrt{|x|}+\sqrt{|y|}+\sqrt{|z|}<1$ and $m<\operatorname{Re} a<\operatorname{Re} e$.

Proof The desired equality follows by a straightforward calculation using Corollary 3.10 in [21], p.12 and Definition 2.4.

To present another application of the above results, we recall some well-known facts. We have Djrbashian's Cauchy type kernel [22], p.76:

$$
C_{\omega}(z)=\sum_{k=0}^{+\infty} \frac{z^{k}}{\Delta_{k}}, \quad \Delta_{0}=1, \Delta_{k}=k \int_{0}^{1} t^{k-1} \omega(t) d t, k \in \mathbb{N} .
$$

One can see that $C_{\omega}(z)$ is a holomorphic function in the unit disc (we denote this by $\mathbb{D}=$ $\{z \in \mathbb{C}:|z|<1\}$ ) for any $\omega(t) \in \Omega_{0}$ (where $\Omega_{0}$ is the class defined in [22], p.76).

Remark 5.1 For the particular case of power functions $\omega(x)=(1-x)^{\alpha},-1<\alpha<0, C_{\omega}(z)$ is the $1+\alpha$ order of the ordinary Cauchy kernel:

$$
C_{\omega}(z)=\frac{1}{(1-z)^{1+\alpha}}:=C_{\alpha}(z), \quad \text { and } \quad C_{1}(z)=\frac{1}{1-z}, \quad z \in \mathbb{D} .
$$

Definition 5.1 Let $E \subset[0,2 \pi]$ be a Borel measurable set (B-set) and $\omega \in \Omega_{0}$. It is said that $E$ is of positive $\omega$-capacity $\left(C_{\omega}(E)>0\right)$ if there exists a nonnegative $B$-measure $\mu$ supported and finite on $E$ and such that

$$
S_{1} \equiv \lim _{r \rightarrow 1-0} \max _{0 \leq \varphi \leq 2 \pi} \int_{0}^{2 \pi}\left|C_{\omega}\left(r e^{i(\varphi-\theta)}\right)\right| d \mu(\theta)<+\infty
$$

If there is no such a measure, i.e. if $S_{1}=\infty$ for any nonnegative $B$-measure, then $E$ is said to be of zero $\omega$-capacity $\left(C_{\omega}(E)=0\right)$.

Note that if we take $\omega(x)=(1-x)^{\alpha}(-1<\alpha<0)$, the last definition becomes that of the well-known Frostman $\alpha$-capacity [22].

Corollary 5.3 If $\sigma(\theta)$ is a function of bounded variation on $[0,2 \pi]$, then the function

$$
F_{\alpha}(z)=\frac{1}{2 \pi} \int_{0}^{2 \pi}{ }_{2} F_{1}\left(\alpha+1,1 ; 1 ; z e^{-i \theta} ; 1\right) d \sigma(\theta), \quad-1<\alpha<0,|z|<1,
$$


has non-zero, finite nontangential boundary values $F_{\alpha}\left(e^{i \varphi}\right)$ at all points $\varphi \in[0,2 \pi]$, with a possible exception of a set $S \subset[0,2 \pi]$ of zero $\alpha$-capacity.

Proof First, one can see that

$$
{ }_{2} F_{1}\left(\alpha+1,1 ; 1 ; z e^{-i \theta} ; 1\right)=\frac{1}{\left(1-z e^{-i \theta}\right)^{\alpha+1}}=\omega_{1}\left(z e^{-i \theta}, \alpha\right), \quad \theta \in[0,2 \pi] .
$$

Besides, the function $\omega(x)=(1-x)^{\alpha}(-1<\alpha<0)$ belongs to the class $\Omega_{0}$, and therefore $C\left(z e^{-i \theta} ; \omega\right)$ becomes $C\left(z e^{-i \theta} ; \alpha\right)=\omega_{1}\left(z e^{-i \theta}, \alpha\right)(\theta \in[0,2 \pi])$ by Remark 5.1. Hence, by Theorem 2.5 in [22], p.112, we find that the function $F_{\alpha}(z)$ has non-zero, finite nontangential boundary values at all points $e^{i \varphi}(\varphi \in[0,2 \pi])$, except in a set $S \subset[0,2 \pi]$ of zero $\alpha$ capacity.

On the other hand, if $f$ is a continuous function in $[0, b]$ and $t \in[0, b]$, then $D_{t}^{\mu, 1} f(t)$ (the operator defined in equation (1.2)) becomes ${ }_{0}^{R C} D_{t}^{\mu} f(t)$ (see [23], p.4, equation (10)), where $m-1<\mu<m, m \in \mathbb{N}(\Im \mu=0)$. Thus, if we consider the fractional problem about calculus of variations described in $[23,24]$ with our operator $D_{z}^{\mu, \tau} f(z)$, under the conditions $z \in \mathbb{R}$, $m-1<\mu<m$ and $m \in \mathbb{N}$. We find that the functional

$$
I[p(\cdot)]=\int_{0}^{b} L\left(t, p(t), D_{t}^{\mu, 1} p(t)\right) d t
$$

where $[0, b] \subset \mathbb{R}, 0<b, 0<\mu<1$, and the functions $p(t)$ and the Lagrangian $L$ : $(t, p, v l) \rightarrow L(t, p, v l)$ are considered to be functions of class $C^{2}\left(p(\cdot) \in C^{2}([0, b] ; \mathbb{R})\right.$, $\left.L(\cdot, \cdot, \cdot) \in C^{2}([0, b] \times \mathbb{R} \times \mathbb{R} ; \mathbb{R})\right)$, satisfies the fractional Euler-Lagrange equation in the sense of Riesz-Caputo (see [23, 24]), i.e.

$$
\partial_{2} L\left(t, p(t), D_{t}^{\mu, 1} p(t)\right)-\frac{1}{\Gamma(1-\mu)} \frac{d}{d t} \int_{0}^{t}(t-\theta)^{-\mu}\left(\partial_{3} L\left(\theta, p(\theta), D_{\theta}^{\mu, 1} p(\theta)\right)\right) d \theta=0
$$

where $\partial_{i} L$ is the partial derivative of $L$ with respect to its $i$ th argument $(i=1,2,3)$ and $p(\cdot)$ is an extremizer of the functional $I[p(\cdot)]$ for all $t \in[0, b]$.

\section{Conclusions}

We hope to find some engineering applications related to our new results. Also, we analyze the possibilities to find solutions of partial differential equations or differential equations in terms of our results. Besides, we are trying to write the weighted hypergeomeric functions like Poisson integrals considering some special weights to find boundary values, factorizations of this functions and applications.

Competing interests

The authors declare that they have no competing interests.

Authors' contributions

All authors jointly worked on deriving the results and approved the final manuscript.

\section{Author details}

'Institute of Mathematics, University of Antioquia, Cl. 53-108, Medellin, Colombia. ${ }^{2}$ Department of Mathematics, University Putra Malaysia, Serdang, Selangor 43400 UPM, Malaysia. ${ }^{3}$ Department of Mathematics, Anand International College of Engineering, Jaipur, 303012, India. 


\section{Acknowledgements}

The authors would like to thank the reviewers for careful reading the paper and giving constructive comments, which improved the manuscript substantially. The authors also acknowledge that this work is partially supported by the Universiti Putra Malaysia.

\section{Publisher's Note}

Springer Nature remains neutral with regard to jurisdictional claims in published maps and institutional affiliations.

Received: 30 November 2016 Accepted: 31 March 2017 Published online: 05 April 2017

\section{References}

1. Mainardi, F: Fractional Calculus and Waves in Linear Viscoelasticity: An Introduction to Mathematical Models (2010)

2. Srivastava, HM: A contour integral involving Foxs H-function. Indian J. Math. 14, 1-6 (1972)

3. Agarwal, $P, C$ Chand, $M$, Purohit, SD: A note on generating functions involving the generalized Gauss hypergeometric function. Nat. Acad. Sci. Lett. 37(5), 457-459 (2014)

4. Agarwal, P, Koul, CL: On generating functions. J. Rajasthan Acad. Phys. Sci. 2(3), 173-180 (2003)

5. Ozergin, E: Some properties of hypergeometric functions. Ph.D. thesis, Eastern Mediterranean University, North Cyprus (2011)

6. Srivastava, HM: Certain generating functions of several variables. Z. Angew. Math. Mech. 57, 339-340 (1977)

7. Lee, DM, Rathie, AK, Parmar, R, Kim, YS: Generalization of extended beta function, hypergeometric function and confluent hypergeometric functions. Honam Math. J. 33, 187-206 (2011)

8. Chaudhry, MA, Qadir, A, Rafique, M, Zubair, SM: Extension of Euler's beta function. J. Comput. Appl. Math. 78, 19-32 (1997)

9. He, JH: A tutorial review on fractal spacetime and fractional calculus. Int. J. Theor. Phys. 53, 3698-3718 (2014)

10. Liu, FJ, Li, ZB, Zhang, S, Liu, HY: He's fractional derivative for heat conduction in a fractal medium arising in silkworm cocoon hierarchy. Therm. Sci. 19, 1155-1159 (2015)

11. Yang, XJ, Baleanu, D, Srivastava, HM: Local Fractional Integral Transforms and Their Applications. Academic Press, Amsterdam (2016)

12. Kiymaz, IO, Cetinkaya, A, Agarwal, P: An extension of Caputo fractional derivative operator and its applications. J. Nonlinear Sci. Appl. 9(6), 3611-3621 (2016)

13. Daftardar-Gejji, V, Bhalekar, S: Boundary value problems for multi-term fractional differential equations. J. Math. Anal. Appl. 345, 754-765 (2008)

14. Jain, S, Choi, J, Agarwal, P: Generating functions for the generalized Appell function. Int. J. Math. Anal. 10(1), 1-7 (2016)

15. Anderson, GD, Barnard, RW, Richards, KC, Vamanamurthy, MK, Vuorinen, M: Inequalities for zero-balanced hypergeometric functions. Trans. Am. Math. Soc. 347(5), 1713-1723 (1995)

16. Barnard, R, Richards, K: A note on the hypergeometric mean value. Comput. Methods Funct. Theory 1(1), 81-88 (2001)

17. Karp, D, Sitnik, SM: Inequalities and monotonicity of ratios for generalized hypergeometric function. J. Approx. Theory $161,337-352$ (2009)

18. Chand, M, Agarwal, $\mathrm{P}, \mathrm{Choi}$, J: Note on generating relations associated with the generalized Gauss hypergeometric function. Appl. Math. Sci. 10(35), 1747-1754 (2016)

19. Li, H: Lipschitz spaces and $Q_{K}$ type spaces. Sci. China Math. 53, 771-778 (2010)

20. Saxena, RK, Kalla, SL, Saxena, R: Multivariable analogue of generalized Mittag-Leffler function. Integral Transforms Spec. Funct. 22(7), 533-548 (2011)

21. Chand, M, Guariglia, E: Note on Euler type integrals. Int. Bull. Math. Res. 2(2), 1-17 (2015)

22. Djrbashian, MM, Zakaryan, VS: Classes and Boundary Properties of Functions Meromorphic in the Disc. Nauka, Moscow (1993)

23. Frederico, G, Torres, D: Fractional Noether's theorem in the Riesz Caputo sense. Appl. Math. Comput. 217, 1023-1033 (2010)

24. Agrawal, OP: Fractional variational calculus in terms of Riesz fractional derivatives. J. Phys. A 40(24), 6287-6303 (2007)

\section{Submit your manuscript to a SpringerOpen ${ }^{\ominus}$ journal and benefit from:}

- Convenient online submission

- Rigorous peer review

- Immediate publication on acceptance

Open access: articles freely available online

High visibility within the field

- Retaining the copyright to your article 